\title{
Classification of Faults on Transmission lines using EMTP and Wavelet Multiresolution Analysis
}

\author{
Mandira Baruah $^{1}$, Mrs. Purabi Patowary ${ }^{2}$ \\ ${ }^{1}$ Student,M.E., ${ }^{2}$ Associate Professor Department of Electrical \& Instrumentation Engineering Assam \\ Engineering College, Guwahati-781013,Assam
}

\begin{abstract}
This paper presents a technique for classification of faults on single circuit transmission line using Wavelet Multiresolution Analysis.EMTP (Microtran) is used to perform simulations which generate time domain input signal on a $400 \mathrm{KV}, 300 \mathrm{~km}$ transmission line fed from one end. Daubechies eight (D-8)wavelet transforms of the three phase currents on a transmission line fed from one end are used for fault analysis in this work. The summation of the $1^{\text {st }}$ level output of MRA detail signals of current in each phase extracted from the original signals generated are employed in fault classification algorithm. Simulation results show that the proposed method is effective and simple and give accurate results irrespective of fault location, fault inception angle and fault impedance.
\end{abstract}

Keywords: Wavelet transforms, Multiresolution analysis, EMTP, fault classification

\section{Introduction}

Faults on transmission line are caused by factors such as lightning strikes, flashover on contaminated insulator surface, broken conducting lines, short circuit between conducting lines, etc. Occurrences of faults in transmission lines give rise to electromagnetic transients in power systems. A fault occurs when two or more conductors come in contact with each other or ground in three phase systems. Faults are classified as Single lineto-ground (L-G) faults, Line-to-line (L-L) faults, Double line-to-ground (LL-G) faults, and three phase (3- $\varphi$ ) faults. The power system components suffer excessive stresses due to extremely high magnitude currents as a result of such transient phenomena. These faults give rise to serious damage on power system equipment. Faults which occur on transmission lines affects both the equipment and the power quality. So, it is necessary to determine the fault type and location on the line and clear the fault as soon as possible in order to avoid such damages.

During 1980s, there were several techniques used to detect and classify the faults on transmission lines such as the variation of the voltage and current of the three phases [1], the ratio of the change in the magnitude of the current to threshold value. During 1990s, Artificial Intelligence techniques were used in power systems and its application in transient analysis has been increased in recent years[5]. FFT (Fast Fourier Transform) is also a useful technique for fault classification but it has poor time localization property for high frequency components of the signal. This shortcoming can be removed to a large extent by using short time Fourier transform (STFT) but it does not provide multiple resolutions in time and frequency as the window size is fixed and hence it is inefficient for accurate analysis of localized transient structure [3]. Wavelets were first applied to power system transients in 1994 by Robertson, Camps and Mayer [2] though O.A.S Youssef [4] initially proposed wavelet transform for fault classification. In recent years, a lot of techniques like wavelet multiresolution analysis has been used in combination with other methods in fault analysis[6]-[10]. D.Chanda et al used DOS-based version of EMTP software and wavelet theory for his extensive work on transient analysis[11].

Wavelet multiresolution analysis is devoid of many shortcomings as it uses short time intervals for high frequency components and long time intervals for low frequency components. In this method, the time domain signal is decomposed into various 'levels' or 'scales' using 'mother wavelet' for analysis.

The presented work uses Wavelet Multiresolution Analysis (MRA) technique for fault classification. The $1^{\text {st }}$ level MRA detail signal is considered and used for the fault analysis. The presented technique does not depend on any threshold value of any of the parameters taken into consideration for the analysis. The EMTP software used in this work is MicroTran v3.11 which is a WINDOWS-based version. As this version limits the size of the power system network to 18 nodes and 12 branches, a $400 \mathrm{KV}, 300 \mathrm{~km}$ transmission line fed from one end is considered. For modeling large power systems, the higher versions of the software are applicable. Review of literature makes no claim for exhaustiveness but only draws upon certain points which have a direct or indirect bearing upon studies undertaken in this work and the motivation for it. 


\section{An Overview Of Emtp}

Microtran v 3.11 is the EMTP software used for the transient analysis. Presently, the EMTP and other programs that are built on a kernel (such as electromagnetic transients) for DC (EMTDC) and power system computer-aided design (PSCAD) based on the same principles are widely used and accepted programs for the computation of electrical transients in power systems. The developers of the EMTP chose methods which they felt are best suited for a general-purpose program such as the EMTP. For analyzing specific problems, other methods may well be competitive, or even better. For example, Fourier transformation methods may be preferable for studying wave distortion and attenuation along a transmission line in cases where the time span of the study is so short that reflected waves have not yet come back from the remote end. The EMTP has been specifically developed for power system problems, but some of the methods have applications in electronic circuit analysis as well. Digital computers cannot simulate transient phenomena continuously, but only at discrete intervals of time. This leads to truncation errors which may accumulate from step to step and cause divergence from the true solution. Most methods used in the EMTP are numerically stable and avoid this type of error build-up. The solution method used in EMTP is based on the phenomenon of travelling waves along a transmission line which are initiated when transients occur. The general solution of the travelling wave equation for a lossless transmission line is given by

$$
\mathrm{v}(\mathrm{x}, \mathrm{t})=\mathrm{v}_{\mathrm{f}}(\mathrm{x}-\mathrm{vt})+\mathrm{v}_{\mathrm{b}}(\mathrm{x}+\mathrm{vt})
$$

where $\mathrm{v}=1 / \sqrt{\mathrm{LC}}=$ speed of waves in $\mathrm{m} / \mathrm{s} ., \mathrm{v}_{\mathrm{f}}$ is the voltage wave which travels in the forward(positive) direction of $\mathrm{x}$ and $\mathrm{v}_{\mathrm{b}}$ travels in the backward direction , $\mathrm{x}=$ length of the transmission line, $\mathrm{t}=$ time. The EMTP can solve any network which consists of interconnections of resistances, inductances, capacitances, single and multiphase $\pi$-circuits, distributed-parameter lines and certain other elements.

\section{Wavelet Multiresolution Analysis}

Wavelet is a wave form of effectively limited duration with an average value of zero. Wavelet is asymmetric and is of irregular wave. Sharp changes which are found in transient signals can be better analyzed with an irregular wavelet rather than using a smooth sinusoid. A wavelet, in the sense of the Discrete Wavelet Transform (DWT) which is being used in this work, is an orthonormal function which can be applied to a finite group of data. It provides multiple resolutions in both time and frequency which is the reason behind its successful implementation in transient analysis. Wavelet analysis, however, can easily resolve signals of nonstationary nature unlike others. Time domain information is retained in the wavelet transform whereas it may be lost in other integral transform techniques such as Fourier analysis. The advantage of using Wavelet analysis is the application of multiresolution property of time-frequency (time-scale). Wavelet analysis is the breaking up of a signal into shifted and scaled version of the original (or mother) wavelet. Scaling a wavelet means stretching (or compressing) it. Shifting a wavelet simply means delaying its onset. It is the orthogonality property of some wavelets which makes it suitable for the study of power sytem dynamics. The analyzing wavelets are called the 'mother wavelets' and its dilated and translated versions are called the 'daughter wavelets'. The choice of 'mother wavelet' involves a prior knowledge of the solution so that the calculations are optimized in some sense. The daubechies Wavelets are able to accurately reconstruct different types of disturbances in the power system as compared to other types of wavelets, so daubechies wavelet is chosen for the analysis. In this work, Daubechies- 8 wavelet is chosen as the 'mother wavelet' as it closely matches the signal to be processed which is of utmost importance in wavelet applications. Daubechies- 8 wavelet is comparatively more compactly supported in time and hence is effective for short and fast transient analysis and provides almost perfect reconstruction. Daubechies- 8 wavelet is smooth and oscillatory in nature which resembles the nature of the transient signals and hence is more suitable for representing the signals.

\section{Wavelet Multi Resolution Analyis For Fault Classification}

By using wavelets, the sub band information regarding different harmonics can be extracted from the original signal. These informations provide the characteristic features of the fault signals and hence the faults can be classified. Simulations are carried out on the transmission line considered for the analysis using Electromagnetic Transients Program (MicroTran) by changing the location of fault on the transmission line. The generated time domain signals in each case are analyzed through Wavelet transform using MATLAB 2009b. The signals in each case have been decomposed into 11 wavelet levels using D- 8 wavelet and first level wavelet output has been considered for the analysis as this level includes the harmonic components most prominently present in fault current signals. As the sampling time considered in the analysis is $10 \mu \mathrm{s}$, which corresponds to a sampling frequency of $100 \mathrm{kHz}$ and the total number of wavelet levels considered is 11 , therefore, 11th level wavelet output corresponds to a frequency band of 50-100 kHz. Downsampling by two at each succeeding level (10th-1st level) will lead to a first level output corresponding to a frequency band of 97-195 $\mathrm{Hz}$ i.e.it includes 2nd and 3rd harmonic components, which are predominant in case of faults. 


\section{Fault Cases Study And Results}

The model power system considered in the analysis is as shown in Fig 4.1. The base values of the voltage and the power in the system are taken as $400 \mathrm{KV}$ and $1000 \mathrm{MVA}$. The frequency of the system is taken as $50 \mathrm{~Hz}$. The sequence parameter line model is selected for the transmission line as the Microtran v 3.1 accepts this model to be suitable in the event of any type of abnormal conditions in transmission lines.

The transmission line parameters are-

(1)Zero sequence parameters:

$\mathrm{R}_{0}=0.3885 \Omega / \mathrm{km}, \mathrm{X}_{0}=3.2510 \mathrm{mH} / \mathrm{km}, \mathrm{C}_{0}=0.0085 \mu \mathrm{F} / \mathrm{km}$;

(2)Positive sequence parameters

(3)Negative sequence parameters:

$$
\mathrm{R}_{1}=0.0233 \Omega / \mathrm{km}, \mathrm{X}_{1}=0.9510 \mathrm{mH} / \mathrm{km}, \mathrm{C}_{1}=0.0124 \mu \mathrm{F} / \mathrm{km}
$$

Same as positive sequence parameters

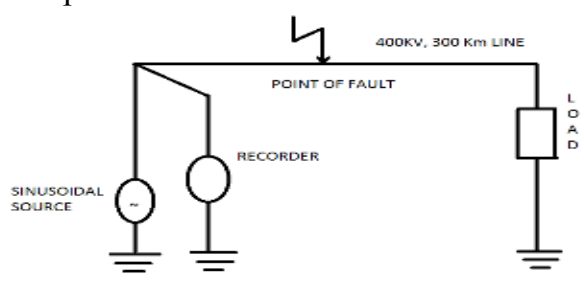

Fig1. Transmission line system used for simulation studies

The total impedance of the generator and the transformer taken together is $(0.2+\mathrm{j} 4.49) \Omega$ which corresponds to an $\mathrm{X} / \mathrm{R}$ ratio of 22.45 . The total impedance considered is $(720+\mathrm{j} 1111) \Omega$, corresponding to a load of $200 \mathrm{MVA}$ at a power factor of 0.9 lag. The current signals recorded at the generator end considered for the analysis are generated by simulating the system on EMTP. The generated time domain signals are sampled every $10 \mu \mathrm{s}$ and then used for the analysis using wavelet transform. The fault resistance is taken as $0.04 \Omega$.

We have considered single line to ground (L-G), double line to ground (LL-G), double line (L-L), and three phase symmetrical (3- $\varphi$ ) faults. The current waveforms for the considered system for each type of fault, generated using EMTP are shown below:

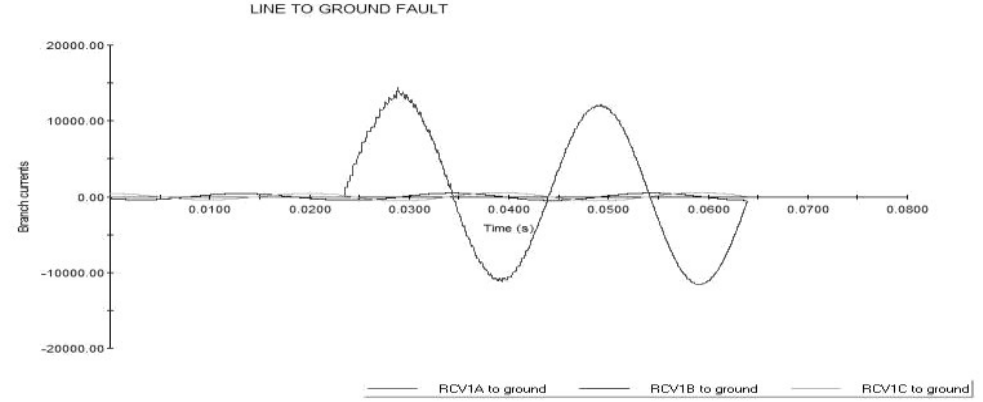

Fig.2 Time domain current signal for single L-G in phase 'a' at dist of $50 \mathrm{~km}$.

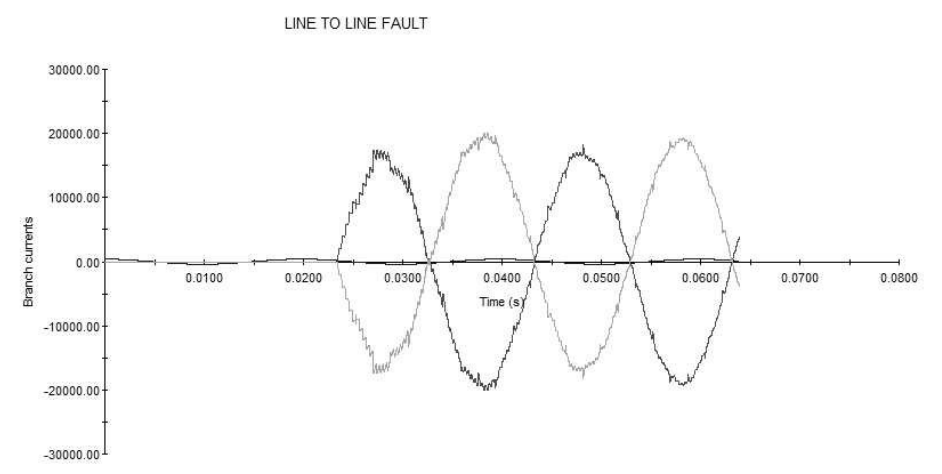

- RCYAAto RCYB - RCVICto ground - RCY1B to RCYAA

Fig.3 Time domain current signals for L-L fault involving phase 'a' and phase 'b' at dist of $50 \mathrm{~km}$. 


\section{LINE TO LINE TO GROUND FAULT}

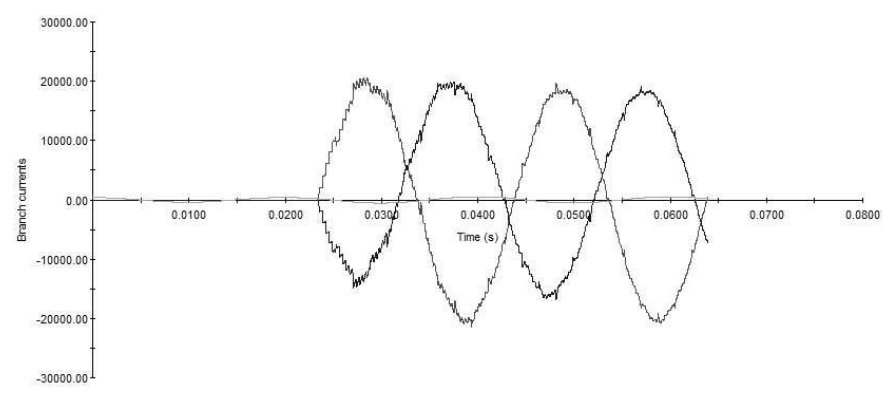

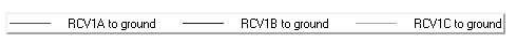

Fig.4 Time domain current signals for LL-G fault involving phase 'a' and phase ' $b$ ' at dist of $50 \mathrm{~km}$.

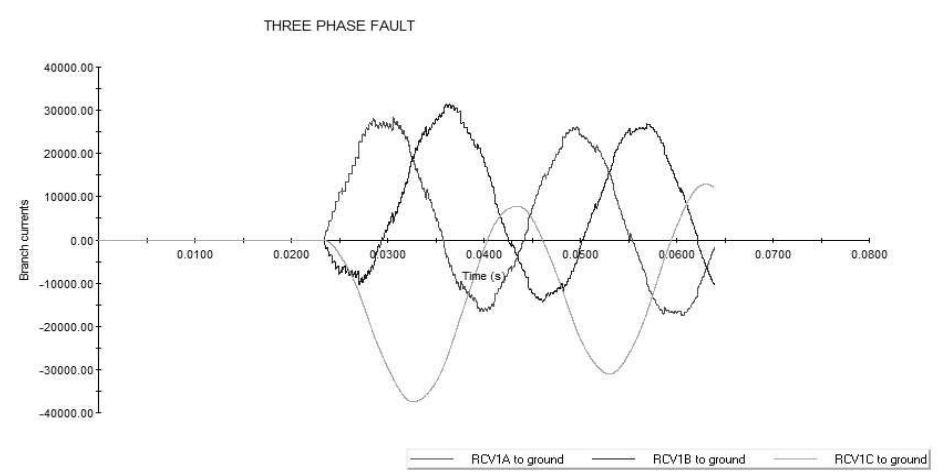

Fig.5 Time domain current signals for 3- $\varphi$ fault at dist of $50 \mathrm{~km}$.

By changing the loacation of fault on the transmission line, simulations are carried out. The data considered in the analysis is assumed to be of finite duration and of length $2^{\mathrm{N}}$, where $\mathrm{N}$ is an integer. If $\mathrm{N}$ is chosen to be 10 , the total duration of the analysis comes out to be $2^{10}$ i.e. $(1024$ X 10$) \mu \mathrm{s}=10.24 \mathrm{~ms}$ which is sufficient enough for the analysis of faults. With $\mathrm{N}=10$, there are $(\mathrm{N}+1)=10+1=11$ wavelet levels, and if these 11 levels are added together then the original signal is faithfully reproduced at each of the sample points.

\section{Fault Classification}

The types of faults considered in the analysis are L-G, L-L, LL-G, 3- $\varphi$ faults. The wavelet toolbox in MATLAB2009a has been used for DWT operation. Eleven wavelet levels are obtained for each of the fault current signal in each fault case. First level wavelet output is considered for the analysis and the parameter chosen for fault classification is the sum of $1^{\text {st }}$ level wavelet output for the three phase currents.

Let $S_{a}=$ summation of $1^{\text {st }}$ level values for current in phase ' $a$ '

$\mathrm{S}_{\mathrm{b}}=$ summation of $1^{\text {st }}$ level values for current in phase ' $\mathrm{b}$ '

$\mathrm{S}_{\mathrm{c}}=$ summation of $1^{\text {st }}$ level values for current in phase ' $c$ '.

Tables (1 to 4) show the values of $S_{a}, S_{b}, S_{c}$ for L-G, L-L, LL-G, 3- $\varphi$ faults. The results are shown for the various fault locations considered along the transmission line. From these tables it is observed that the magnitudes of $\mathbf{S a}, \mathbf{S b}, \mathbf{S c}$ vary in a particular pattern for a particular type of fault occurring in a transmission line. Based on first level wavelet output, an efficient algorithm is presented. 


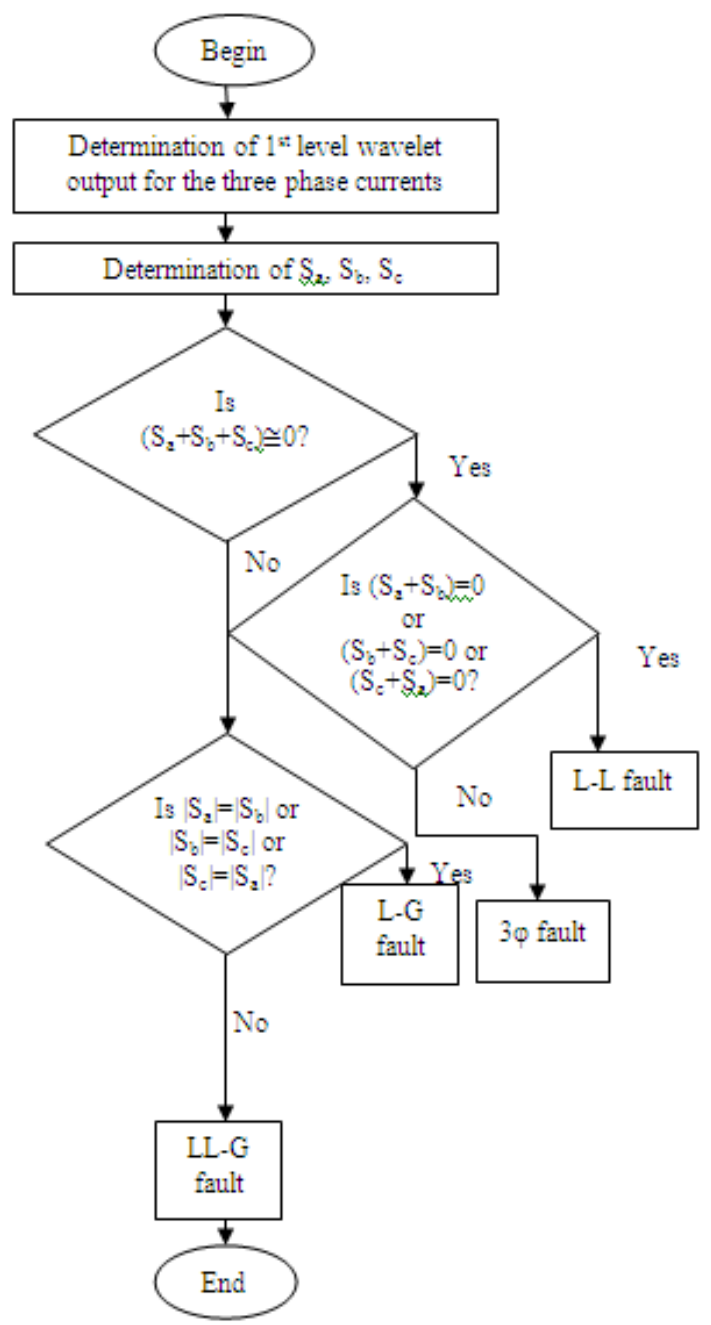

Fig.6 Algorithm for Transmission line fault classification

When the algebraic sum of $\mathbf{S}_{\mathrm{a}}, \mathbf{S}_{\mathrm{b}}, \mathbf{S}_{\mathrm{c}}$ is zero, then it is 3- $\varphi$ fault. The $1^{\text {st }}$ level wavelet output for 3- $\varphi$ fault is shown in Table1. When the algebraic sum of $\mathbf{S}_{\mathrm{a}}, \mathbf{S}_{\mathrm{b}}, \mathbf{S}_{\mathrm{c}}$ is zero and also if sum of any two of the summations $\mathrm{S}_{\mathrm{a}}$, $\mathrm{S}_{\mathrm{b}}, \mathrm{S}_{\mathrm{c}}$ is equal to zero, i.e. the magnitude of one of the summations is almost negligible in comparison to the equal magnitudes of other two summations, then the fault is classified as L-L fault. $\mathrm{S}_{\mathrm{c}}$ is very small in each of the cases for L-L fault in phase ' $a$ ' and phase ' $b$ ' as is evident from Table 2.

Table 1 Values of summation of $1^{\text {st }}$ level output for different points of faults in three phase fault, fault inception

\begin{tabular}{|c|c|c|c|}
\hline $\begin{array}{c}\text { Point of } \\
\text { fault (in } \\
\text { km.) }\end{array}$ & $\mathbf{S}_{\mathbf{a}}$ & $\mathbf{S}_{\mathbf{b}}$ & $\mathbf{S}_{\mathbf{c}}$ \\
\hline 10 & -25.00 & 50.90 & -25.90 \\
\hline 20 & -16.97 & 29.50 & -12.54 \\
\hline 30 & -7.18 & 16.18 & -8.99 \\
\hline 40 & 4.14 & 2.98 & -7.13 \\
\hline 50 & 11.83 & -5.44 & -6.39 \\
\hline 60 & 3.78 & 1.25 & -5.03 \\
\hline 70 & -8.82 & 12.33 & -3.50 \\
\hline 80 & 4.28 & -0.83 & -3.45 \\
\hline 90 & -1.40 & 4.64 & -3.23 \\
\hline 100 & -0.93 & 3.96 & -3.03 \\
\hline 110 & -5.60 & 7.80 & -2.19 \\
\hline 120 & -1.54 & 4.04 & -2.49 \\
\hline 130 & -4.17 & 6.17 & -1.99 \\
\hline 140 & -2.98 & 4.83 & -1.84 \\
\hline 150 & -1.43 & 0.73 & -2.17 \\
\hline 160 & -2.61 & 4.23 & -1.61 \\
\hline
\end{tabular}




\begin{tabular}{|c|c|c|c|}
\hline 170 & -3.20 & 4.73 & -1.53 \\
\hline 180 & -1.03 & 2.70 & -1.67 \\
\hline 190 & -3.25 & 4.53 & -1.28 \\
\hline 200 & -0.47 & 1.99 & -1.52 \\
\hline 210 & -0.60 & 2.00 & -1.39 \\
\hline 220 & 1.57 & -0.30 & -1.26 \\
\hline 230 & -2.70 & 3.79 & -1.08 \\
\hline 240 & 0.95 & 0.31 & -1.27 \\
\hline 250 & 2.39 & -1.10 & -1.29 \\
\hline 260 & 0.64 & 0.46 & -1.11 \\
\hline 270 & -1.10 & 1.53 & -0.29 \\
\hline 280 & -1.22 & 2.14 & -0.91 \\
\hline 290 & -0.87 & 1.78 & -0.91 \\
\hline
\end{tabular}

Table 2 Values of summation of $1^{\text {st }}$ level output for different points of faults in line to line fault at phase ' $a$ ' and phase ' $\mathrm{b}$ ' fault inception angle $=0^{0}$

\begin{tabular}{|c|c|c|c|}
\hline $\begin{array}{l}\text { Point of } \\
\text { fault (in } \\
\text { Km.) }\end{array}$ & $\mathbf{S}_{\mathbf{a}}$ & $\mathbf{S}_{\mathrm{b}}$ & $\mathbf{S}_{\mathrm{c}}$ \\
\hline 10 & -37.58 & 37.58 & 0.10 \\
\hline 20 & -17.22 & 17.22 & 0.10 \\
\hline 30 & -12.63 & 12.63 & 0.10 \\
\hline 40 & 0.45 & -0.45 & 0.10 \\
\hline 50 & 3.76 & -3.76 & 0.10 \\
\hline 60 & -18.60 & 18.60 & 0.09 \\
\hline 70 & -10.05 & 10.05 & 0.09 \\
\hline 80 & 3.36 & -3.36 & 0.09 \\
\hline 90 & -2.30 & 2.30 & 0.09 \\
\hline 100 & -1.49 & 1.49 & 0.09 \\
\hline 110 & -8.17 & 8.17 & 0.09 \\
\hline 120 & -4.03 & 4.03 & 0.09 \\
\hline 130 & -7.21 & 7.21 & 0.08 \\
\hline 140 & -4.81 & 4.81 & 0.08 \\
\hline 150 & -1.40 & 1.40 & 0.08 \\
\hline 160 & -3.64 & 3.64 & 0.08 \\
\hline 170 & 11.17 & -11.17 & 0.08 \\
\hline 180 & 0.67 & -0.67 & 0.08 \\
\hline 190 & 0.38 & -0.38 & 0.08 \\
\hline 200 & -3.66 & 3.66 & 0.08 \\
\hline 210 & -11.26 & 11.26 & 0.07 \\
\hline 220 & 0.23 & -0.23 & 0.07 \\
\hline 230 & 11.94 & -11.94 & 0.07 \\
\hline 240 & -1.75 & 1.75 & 0.07 \\
\hline 250 & 4.84 & -4.84 & 0.07 \\
\hline 260 & -7.48 & 7.48 & 0.07 \\
\hline 270 & 6.80 & -6.80 & 0.07 \\
\hline 280 & 6.65 & -6.65 & 0.07 \\
\hline 290 & -1.91 & 1.91 & 0.07 \\
\hline
\end{tabular}

The fact that the summation of $S_{a}, S_{b}, S_{c}$ are equal or not equal to zero discriminates L-G, LL-G faults from L-L, 3- $\varphi$ faults. If the sum of $S_{a}, S_{b}, S_{c}$ is not equal to zero and the absolute value of any two summations $\left(\mathrm{S}_{\mathrm{a}}, \mathrm{S}_{\mathrm{b}}, \mathrm{S}_{\mathrm{c}}\right)$ is equal and also is always much smaller than the absolute value of the $3^{\text {rd }}$ summation, then it is a $\mathrm{L}-$ $\mathrm{G}$ fault. Values of summation of $1^{\text {st }}$ level wavelet output for L-G fault as shown in Table 3 bears testimony to that. Again, if the sum of $S_{a}, S_{b}, S_{c}$ is not equal to zero and if the absolute value of any two summations $\left(S_{a}, S_{b}\right.$, $S_{c}$ ) are not equal and also are always much higher than the absolute value of the $3^{\text {rd }}$ summation, then it is a LLG fault.

Results for LL-G fault in phase 'a' and phase 'b' are shown in Table 4.

Table 3 Values of summation of $1^{\text {st }}$ level output for different points of faults in single line to ground fault at phase ' $a$ ', fault inception angle $=0^{0}$

\begin{tabular}{|c|c|c|c|}
\hline $\begin{array}{c}\text { Point of } \\
\text { fault ( in } \\
\text { km.) }\end{array}$ & $\mathbf{S}_{\mathbf{a}}$ & $\mathbf{S}_{\mathbf{b}}$ & $\mathbf{S}_{\mathbf{c}}$ \\
\hline 10 & -17.61 & 0.35 & 0.19 \\
\hline 20 & -8.44 & 0.35 & 0.20 \\
\hline 30 & -5.45 & 0.36 & 0.20 \\
\hline 40 & -6.52 & 0.38 & 0.22 \\
\hline 50 & -1.28 & 0.32 & 0.16 \\
\hline
\end{tabular}




\begin{tabular}{|c|c|c|c|}
\hline 60 & -3.19 & 0.32 & 0.16 \\
\hline 70 & -1.85 & 0.35 & 0.18 \\
\hline 80 & -2.9 & 0.32 & 0.15 \\
\hline 90 & -3.24 & 0.33 & 0.13 \\
\hline 100 & -2.5 & 0.36 & 0.19 \\
\hline 110 & -1.25 & 0.35 & 0.18 \\
\hline 120 & -0.46 & 0.36 & 0.19 \\
\hline 130 & -3.67 & 0.35 & 0.19 \\
\hline 140 & -2.97 & 0.35 & 0.18 \\
\hline 150 & -1.25 & 0.35 & 0.19 \\
\hline 160 & -2.01 & 0.38 & 0.20 \\
\hline 170 & 0.96 & 0.32 & 0.14 \\
\hline 180 & -1.71 & 0.36 & 0.17 \\
\hline 190 & 0.87 & 0.35 & 0.18 \\
\hline 200 & -0.50 & 0.33 & 0.14 \\
\hline 210 & -0.50 & 0.32 & 0.13 \\
\hline 220 & -1.73 & 0.34 & 0.15 \\
\hline 230 & -3.46 & 0.35 & 0.19 \\
\hline 240 & -1.75 & 0.35 & 0.15 \\
\hline 250 & -1.85 & 0.34 & 0.15 \\
\hline 260 & -1.34 & 0.34 & 0.13 \\
\hline 270 & -0.90 & 0.34 & 0.13 \\
\hline 280 & -0.74 & 0.35 & 0.14 \\
\hline 290 & -1.68 & 0.40 & 0.19 \\
\hline
\end{tabular}

Table 4 Values of summation of $1^{\text {st }}$ level output for different points of faults at phase ' $\mathrm{a}$ ' and phase ' $\mathrm{b}$ ', fault inception angle $=0^{0}$

in double line to ground fault

\begin{tabular}{|c|c|c|c|}
\hline $\begin{array}{l}\text { Point of } \\
\text { fault (in } \\
\text { Km) }\end{array}$ & $\mathbf{S}_{\mathbf{a}}$ & $S_{b}$ & $\mathbf{S}_{\mathrm{c}}$ \\
\hline 10 & -35.17 & 40.23 & 0.12 \\
\hline 20 & -19.11 & 18.48 & 0.12 \\
\hline 30 & -11.24 & 13.51 & 0.12 \\
\hline 40 & 0.74 & 0.37 & 0.12 \\
\hline 50 & 4.103 & -3.09 & 0.12 \\
\hline 60 & 1.32 & -0.50 & 0.11 \\
\hline 70 & -9.64 & 10.33 & 0.11 \\
\hline 80 & 2.83 & -2.22 & 0.11 \\
\hline 90 & -2.08 & 2.55 & 0.11 \\
\hline 100 & -1.29 & 1.72 & 0.11 \\
\hline 110 & -7.91 & 8.35 & 0.10 \\
\hline 120 & -3.82 & 4.22 & 0.10 \\
\hline 130 & -7.03 & 7.36 & 0.10 \\
\hline 140 & -4.63 & 4.98 & 0.10 \\
\hline 150 & -1.26 & 1.55 & 0.10 \\
\hline 160 & -3.55 & 3.79 & 0.10 \\
\hline 170 & 11.21 & -10.98 & 0.09 \\
\hline 180 & 0.80 & -0.50 & 0.09 \\
\hline 190 & 0.48 & -0.25 & 0.09 \\
\hline 200 & -3.52 & 3.76 & 0.09 \\
\hline 210 & -11.05 & 11.30 & 0.09 \\
\hline 220 & 0.34 & -0.11 & 0.08 \\
\hline 230 & 11.89 & -11.69 & 0.08 \\
\hline 240 & -1.68 & 1.85 & 0.08 \\
\hline 250 & 4.94 & -4.75 & 0.08 \\
\hline 260 & -7.28 & 7.40 & 0.08 \\
\hline 270 & 6.40 & -6.23 & 0.08 \\
\hline 280 & 6.27 & -6.18 & 0.08 \\
\hline 290 & -1.85 & 1.97 & 0.07 \\
\hline
\end{tabular}

VII. Conclusion

In this paper, an efficient technique using wavelet multiresolution analysis is presented for classification of faults on a power transmission line. Wavelet multiresolution analysis is found to be most suitable for extracting the information from transient fault signals. The presented technique gives accurate results irrespective of fault location, fault inception angle and fault impedance. As the technique is simple and generalized, it can be used for analysis of high impedance faults. The technique can be applied to transmission lines at any voltage level. 


\section{References}

[1]. D. Breingan, M.M Chen and T.F. Gallen, "Laboratory Investigation for a Digital System for the Protection of Transmission Lines" IEEE Transaction on Power Apparatus and Systems, Vol. PAS 98, No. 2, March/April 1979, pp. 350-368.

[2]. D.C.Robertson, O..Camps, and J.Mayer, "Wavelets and Power System Transients", SPIE International Symposium on Optical Engineering in Aerospace Sensing, 2242:474-87, April 1994.

[3]. Bo Z.Q, F Jiang, Z. Chen, X. Z. Dong, G. Weller, M. A. Redfern, "Transient based protection for power transmission systems," IEEE Power Engineering Society Winter Meeting, vol. 3, pp. 1832-1837, 2000.

[4]. O.A.S.Youssef "Fault classification based on wavelet transforms", IEEE/PES Transmission and Distribution Conference and Exposition, vol.1,pp.531-538,2001.

[5]. Darte G. Cesar, Vega. G. Valdomiro and Ordonez P. Gabriel, “Automatic Power Quality Disturbances Detection and Classification Based on Discrete Wavelet Transform and Artificial Intelligence," IEEE/PES Transmission and Distribution Conf. and Exposition: Latin America 2006, Aug. 2006, pp. 1-6.

[6]. P. Chiradeja and C. Pothisarn "Identification of the Fault Location for Three- Terminal Transmission Lines using Discrete Wavelet Transforms" IEEE T\&D Asia 2009.

[7]. S.A.Shaaban and Prof.Takashi Hiyama, "Transmission Line Faults Classification Using Wavelet Transform" Proceedings of the 14th International Middle East Power Systems Conference (MEPCON'10), Cairo University, Egypt, December 19-21, 2010, Paper ID 225.

[8]. Zhengyou He, Ling Fu, Sheng Lin, and Zhiqian Bo "Fault Detection and Classification in EHV Transmission Line Based on Wavelet Singular Entropy" IEEE Transactions On Power Delivery, Vol. 25, No. 4, October 2010.

[9]. F. B. Costa, B. A. Souza, and N. S. D. Brito, "Realtime detection of fault-induced transients in transmission lines," IET Electronics Letters, pp. 753-755, May 2010.

[10]. A. Jamehbozorg and S. M. Shahrtash "A Decision Tree-Based Method for Fault Classification in Double-Circuit Transmission Lines" IEEE Transactions On Power Delivery, Vol. 25, No. 4, October 2010.

[11]. D. Chanda, N.K. Kishore, A.K. Sinha, "Classification of lightning and switching transients using Wavelet Multiresolution analysis and estimation of waveshapes using Artificial Neural Network on EHV transmission systems" Published in Proceedings of International Conference on Computer applications in Electrical Engineering-CERA 01, Feb.,2002 at Roorkie, India.

[12]. Hermann W. Dommel,EMTP Theory Book, Bonneville Power Administration, United States of America,1987.

[13]. Michel Misiti,Yves Misiti,Georges Oppenheim,Jean Michel Poggi,Wavelet Toolbox User's Guide, The Math Work, Inc, 2001. 